\title{
Precision Microwave Spectroscopy of the Positronium $n=2$ Fine Structure
}

\author{
L. Gurung, T. J. Babij, S. D. Hogan, and D. B. Cassidy \\ Department of Physics and Astronomy, University College London, Gower Street, London WClE 6BT, United Kingdom
}

(Received 25 April 2020; accepted 8 July 2020; published 12 August 2020)

\begin{abstract}
We report a new measurement of the positronium (Ps) $2^{3} S_{1} \rightarrow 2^{3} P_{0}$ interval. Slow Ps atoms, optically excited to the radiatively metastable $2^{3} S_{1}$ level, flew through a microwave radiation field tuned to drive the transition to the short-lived $2^{3} P_{0}$ level, which was detected via the time spectrum of subsequent ground state Ps annihilation radiation. After accounting for Zeeman shifts we obtain a transition frequency $\nu_{0}=18501.02 \pm 0.61 \mathrm{MHz}$, which is not in agreement with the theoretical value of $\nu_{0}=18498.25 \pm 0.08 \mathrm{MHz}$.

DOI: 10.1103/PhysRevLett.125.073002
\end{abstract}

A growing number of observations have arisen that do not seem to fit into the accepted paradigms of modern physics. These phenomena exist in diverse regimes and include the nature of dark matter and dark energy, the origin of $C P$ violation, the mechanisms behind neutrino oscillations, and the apparent lack of antimatter in the Universe. It is not clear if these are manifestations of some single underlying process, or if they defy explanation simply as a consequence of the approximate nature of the standard model (SM) [1]. Therefore, while it is possible that such apparent anomalies can eventually be understood within the existing SM (e.g., Ref. [2]), there is nevertheless a strong desire to search for new physics beyond this framework. This search is increasingly taking place in arenas other than high-energy particle accelerators and, in particular, atomic and molecular techniques are being brought to bear on the problem [3].

At the same time, developments in positron beam technology [4] have opened up new possibilities for Ps studies [5]. As it is composed only of leptons, Ps may be considered to be a pure QED system [6,7]; the low electron and positron mass means that weak force contributions to Ps energy levels are negligible [8], and that Ps is so well described by bound-state QED theory that measured deviations from theoretical predictions can be interpreted as possible indications of new physics. Any such measurements, however, must necessarily be performed with a precision comparable to the corresponding theoretical uncertainties.

All $\mathcal{O}\left(\mathrm{m} \alpha^{6}\right)$ QED corrections to Ps energy levels have been calculated [9-13], with ongoing work on the $\mathcal{O}\left(\mathrm{m} \alpha^{7}\right)$ terms [14-21]. The $n=2$ fine structure intervals have a theoretical uncertainty of $80 \mathrm{kHz}$ (based on estimates of uncalculated terms [10]), while the corresponding experimental uncertainties have been several $\mathrm{MHz}$ for over 25 years [22].

Previous experimental QED tests involving Ps include measurements of the ground state singlet [23] and triplet
[24,25] decay rates, the $1{ }^{3} S_{1} \rightarrow 1{ }^{1} S_{0}$ [26-29] and $1{ }^{3} S_{1} \rightarrow$ $2^{3} S_{1}$ [30-33] intervals, and the $n=2$ fine structure [34-37]. Trap-based positron beams [4] and new laser technology [38] can now be used to improve the precision of such measurements, which are potentially sensitive to new particles or forces (e.g., Refs. [39-42]).

Here we report a new measurement of the $2^{3} S_{1} \rightarrow 2^{3} P_{0}$ interval (designated $\nu_{0}$ ), performed using low energy Ps atoms optically excited to the $2^{3} S_{1}$ level [43]. The apparatus and data analysis methods used in this work are similar to those described elsewhere [44,45]; a pulsed beam containing $\approx 10^{6}$ positrons/pulse with a Gaussian spatial (temporal) profile of approximately $2 \mathrm{~mm}$ (3 ns) (FWHM) was generated using a two-stage Surko-type buffer gas trap [4] and implanted into a mesoporous $\mathrm{SiO}_{2}$ film [46]. The positron beam was guided by an axial magnetic field $B_{z}$ whose strength could be varied from 20-100 G.

Ps atoms emitted from the silica film were irradiated using a broadband (100 GHz, FWHM) pulsed ultraviolet $(\lambda=243.01 \mathrm{~nm})$ dye laser [44], with light propagating in the $+x$ direction. The laser excitation was performed in an electric field of $5.8 \mathrm{kV} / \mathrm{cm}$, which mixed $n=2$ levels with differing orbital angular momentum quantum numbers $\ell$ via the Stark effect, producing $n=2$ states with both $S$ $(\ell=0)$ and $P(\ell=1)$ character. Turning off the electric field immediately after excitation with a fast high-voltage switch allowed $\approx 10 \%$ of the excited states to adiabatically evolve into pure long-lived $2^{3} S_{1}$ atoms [43], which then flew through a microwave guide, as indicated in Fig. 1(a).

A rectangular waveguide with WR51 dimensions $(12.95 \times 6.48 \mathrm{~mm})$ was used. The open sides through which the positron beam and Ps atoms passed were made from high transmission (95\%) tungsten grids. Microwave radiation was coupled into the vacuum system via a highfrequency $(45 \mathrm{GHz}) \mathrm{UHV}$ feed through, and introduced into the waveguide by an antenna, with $\mathrm{TE}_{10}$ microwave radiation propagating along the $-x$ direction, as indicated 


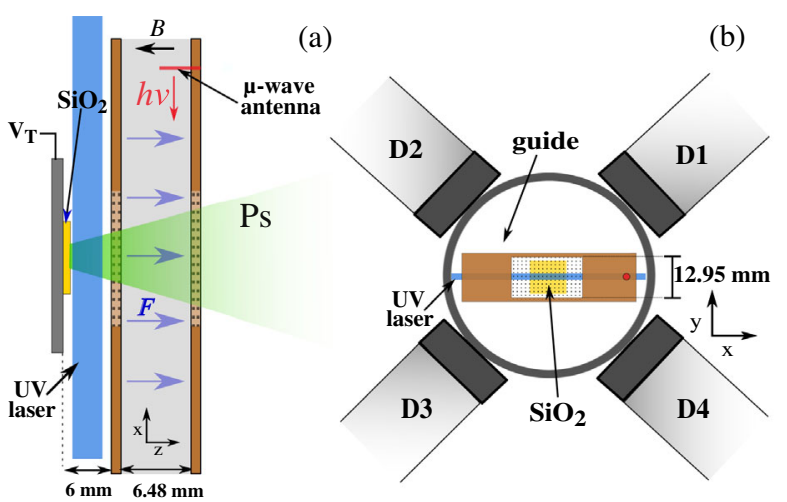

FIG. 1. (a) Schematic of the positronium formation and excitation region and WR-51 waveguide and (b) the positions of the four LYSO detectors D1-4. The spatial profile of the UV excitation laser light is indicated by the (blue) rectangle in (a). $F$ and $B$ represent the electric field of the microwave radiation, and the externally applied magnetic field, respectively.

in Fig. 1(a). Measurements were performed using $\approx 6.5 \mathrm{~mW}$ of microwave power, where the signal generator output was adjusted to provide a constant level at the waveguide input for all frequencies. The bandwidth and absolute frequency calibration of the microwave source were both sub $\mathrm{kHz}$, and entirely negligible in the present experiments.

The $2^{3} S_{1} \rightarrow 2^{3} P_{0}$ transition was driven using polarized microwave radiation such that only $\Delta M_{J}=0$ transitions were allowed (where $M_{J}$ represents the $z$ projection of the total angular momentum vector $\vec{J}=\vec{\ell}+\vec{S}$, and $\vec{S}$ is the Ps spin vector [47]). Thus, only the $2^{3} S_{1}\left(M_{J}=0\right)$ sublevel was depopulated. The metastable $2^{3} S_{1}$ level does not decay radiatively, but has a mean annihilation lifetime $\tau_{\text {ann }}=1.1 \mu \mathrm{s}$ [48]. If population is transferred to the $2^{3} P_{0}$ level, these atoms will decay to the $1^{3} S_{1}$ ground state with a mean radiative lifetime $\tau_{\text {rad }}=3.2 \mathrm{~ns}$, and then self-annihilation will occur with a mean lifetime of 142 ns [48]; selfannihilation of all $2^{3} P_{J}$ levels is negligible [49]. The natural linewidth of the $2^{3} S_{1} \rightarrow 2^{3} P_{0}$ transition is $1 / 2 \pi \tau_{\text {rad }} \approx$ $50 \mathrm{MHz}$ (FWHM). Spontaneous radiative decay may proceed via $\Delta M_{J}=0$ and $\Delta M_{J}= \pm 1$ channels, as indicated in the inset to Fig. 2. Thus, the effect of driving the $2^{3} S_{1} \rightarrow 2^{3} P_{0}$ microwave transition was to reduce the mean lifetime against annihilation of the $2^{3} S_{1}$ population, which may therefore be detected using positron annihilation lifetime spectroscopy.

Ps lifetime spectra were measured using a single-shot technique [50] in which the time profile of Ps annihilation radiation was recorded by four lutetium-yttrium oxyorthosilicate (LYSO) based gamma-ray detectors [45], placed around the vacuum chamber as indicated in Fig. 1(b). Lifetime spectra were parametrized using the quantity $f_{d}=\int_{B}^{C} V(t) d t / \int_{A}^{C} V(t) d t$, where $V(t)$ represents the detector output voltage and $(A, B, C)=(-30,600,1400) \mathrm{ns}$ define the analysis time windows used [44]. Radiation induced changes to Ps decay rates were characterized by the parameter $S_{\gamma}=\left[f_{d}(\right.$ off $)-f_{d}($ on $\left.)\right] / f_{d}($ off $)$, where (on) and (off) refer to the microwave radiation.

Spectra of the $2^{3} S_{1} \rightarrow 2^{3} P_{0}$ transition were obtained by measuring $S_{\gamma}$ as a function of the microwave frequency. The data were corrected according to the frequency dependence of the electric field intensity and microwave power in a waveguide, as described by Hagena et al. [36], although for the waveguide used here this had a negligible effect. Lorentzian functions, using instrumental error weighting, were used to fit the data and obtain the transition frequencies. Although more complicated line shapes can be used [35], for symmetric line shapes any suitable symmetric function (including Lorentzian profiles) will give the same center frequencies [36].

In the absence of broadening, or other perturbing mechanisms, one would expect to observe a Lorentzian profile with a FWHM equal to the $50 \mathrm{MHz}$ natural linewidth. In fact, broader lines were observed, an example of which is shown in Fig. 2. The linewidth averaged over all measurements was $\Gamma_{0}=66 \pm 1 \mathrm{MHz}$; we attribute this to transit time broadening [51], caused by the Ps flight time through the waveguide. Time-of-flight spectra of excited-state Ps generated from silica films have been measured [52], yielding a mean longitudinal Ps speed of $v_{z} \approx 10^{7} \mathrm{~cm} / \mathrm{s}$, with a broad distribution $( \pm 30 \%)$. Slower atoms from this distribution, which are more likely to contribute to the $2^{3} S_{1} \rightarrow 2^{3} P_{0}$ signal, will take approximately $100 \mathrm{~ns}$ to pass through the waveguide; this is sufficient to broaden the linewidths to the observed values. The transverse velocity distribution of the excited state Ps along the $x$ direction was determined by the $100 \mathrm{GHz}$ excitation laser bandwidth, broadening the line by less than

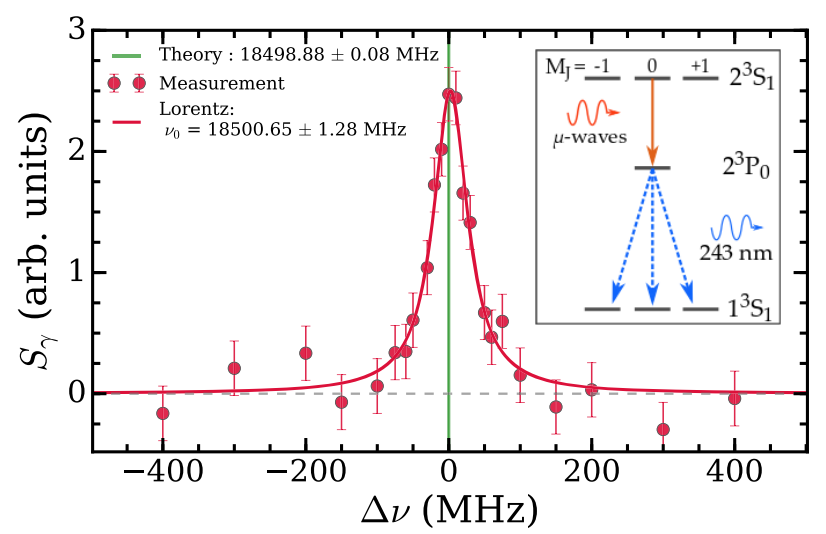

FIG. 2. Example line shape and Lorentzian fit for the $\nu_{0}$ transition measured in a magnetic field of $32 \mathrm{G}$ using detector D2. The vertical line at $\Delta \nu=0$ corresponds to the calculated transition frequency, including the Zeeman shift. The inset shows the microwave (solid arrow) and optical (dashed arrows) transitions by which $2^{3} S_{1}$ atoms reach the $1^{3} S_{1}$ ground state. 


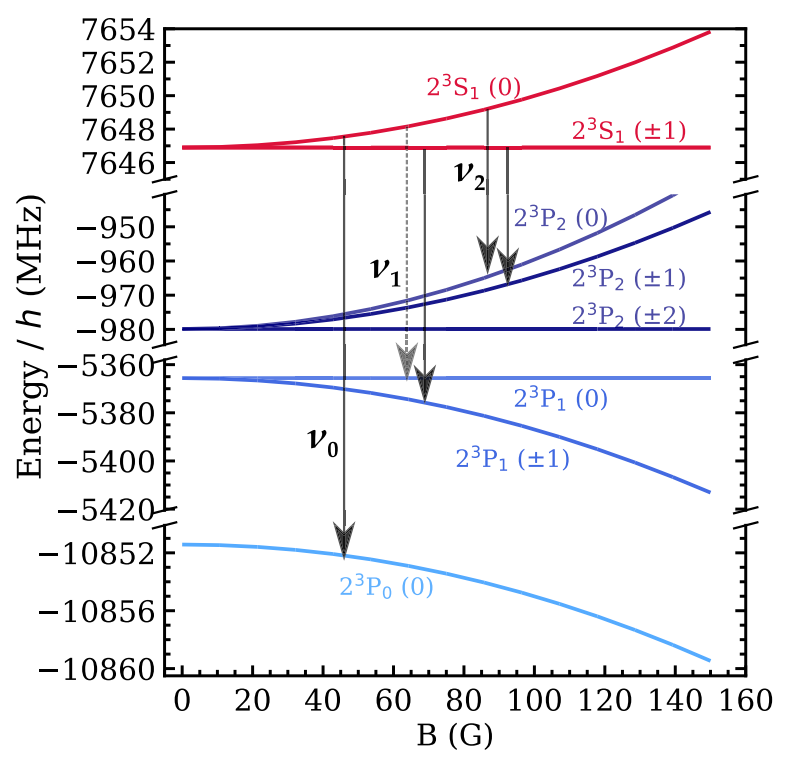

FIG. 3. Calculated Zeeman shifts of $n=2$ triplet Ps levels. The allowed $\nu_{J}$ transitions with $\Delta M_{J}=0$ are indicated by the vertical arrows. The energies shown are relative to the $n=2$ Bohr [i.e., $\left.\mathcal{O}\left(m \alpha^{2}\right)\right]$ level. The $2^{3} S_{1}(0) \rightarrow 2^{3} P_{1}(0)$ transition is shown with a dashed line because the transition strength is zero. Here we designate levels with principal quantum number $n$ using the standard spectroscopic notation $n^{(2 S+1)} \ell_{J}\left(M_{J}\right)$.

$3 \mathrm{MHz}$. This velocity selection also ensures a symmetric transverse angular distribution.

The experiments we describe were conducted in an axial magnetic field, and Zeeman shifts of the $n=2$ energy levels therefore had to be taken into account. The expected shifts have been calculated [49], as shown in Fig. 3. Also indicated in this figure are the allowed $\Delta M_{J}=0$ transitions between (triplet) $n=2$ levels. Measurements were made in a range of magnetic fields, as shown in Fig. 4. These data show the variations in the measured transition frequencies in different magnetic fields (averaged over all four $\gamma$-ray detectors) as well as a quadratic fit, extrapolated to zero magnetic field, and the calculated transition frequencies. The measured linewidths were found to be essentially independent of the magnetic field strength, as shown in Fig. 4(b). The magnetic field in the waveguide was measured with a Hall probe, accurate to $1 \%$. The maximum variations in the magnetic field over the spatial extent of the waveguide were found to be less than $\pm 1 \mathrm{G}$, which was used as the field error in the quadratic fit shown in Fig. 4(a). The extrapolation to zero magnetic field yielded a transition frequency of $18501.02 \pm 0.57 \mathrm{MHz}$, where the error is due to the errors in the individual frequency measurements, and is taken to represent the statistical error of the measurement.

The symmetry of the observed line shapes was verified by fitting Fano profiles $[53,54]$ to the data. These profiles, which can be used to characterize line shapes via an asymmetry parameter $q$, are given by $F(\varepsilon) \propto(q+\varepsilon)^{2} /$ $\left[\left(1+q^{2}\right)\left(1+\varepsilon^{2}\right)\right]$, where the reduced frequency

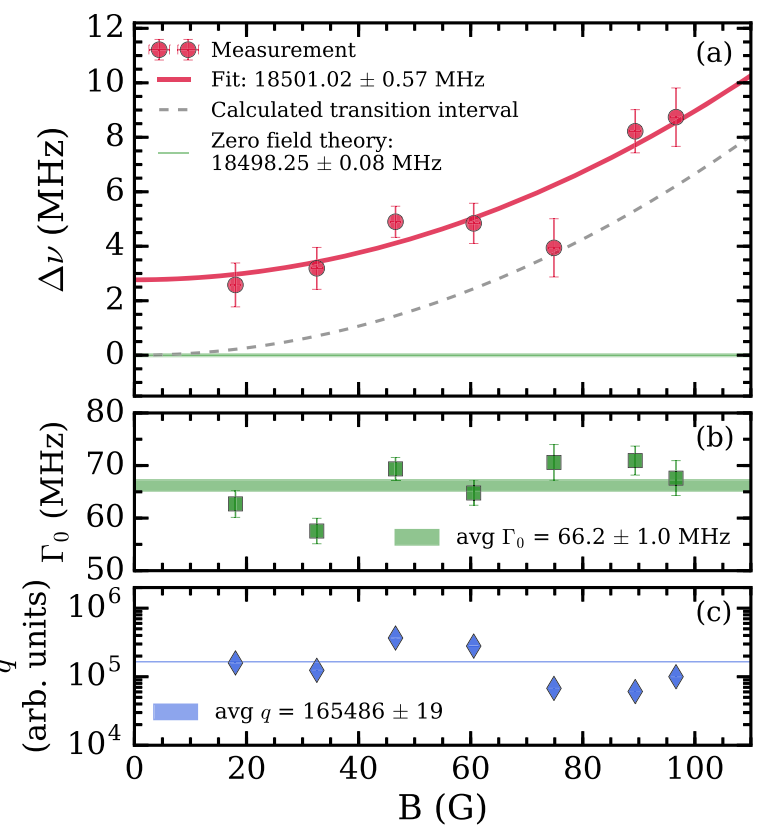

FIG. 4. $2^{3} S_{1} \rightarrow 2^{3} P_{0}$ transition frequency shifts (a), linewidths (b), and Fano asymmetry parameters $q$ (c) measured in different magnetic fields. The dashed (gray) curve in (a) is the calculated Zeeman shift of the $\nu_{0}$ transition frequency (see Fig. 3), and the solid (red) curve is a quadratic fit to the data. These curves have the form $y=a B^{2}+c$. For the calculated curve the coefficient $a=6.7 \times 10^{-4} \mathrm{MHz} / \mathrm{G}^{2}$, and from the fit we obtain $a=(6.2 \pm 1.2) \times 10^{-4} \mathrm{MHz} / \mathrm{G}^{2}$. The data were averaged over all 4 detectors and multiple runs, with a total acquisition live time of $300 \mathrm{~h}$.

$\varepsilon=2\left(\nu-\nu_{R}\right) / \Gamma_{0}, \nu_{R}$ is the resonance frequency and $\Gamma_{0}$ is the corresponding (Lorentzian) FWHM. For large values of $q$ this is equivalent to a Lorentzian; the $q$ values obtained were on the order of $10^{5}$ [see Fig. 4(c)], which are large enough to justify the use of Lorentzian line shapes.

Ps $n=2$ fine structure intervals have been measured in previous experiments, at Brandeis [34], Michigan [35,37], and Mainz [36]. All of these experiments were performed using metal targets that directly produce Ps atoms in $n=2$ states following positron implantation [55-59]. This technique has the advantage that it generates excited state atoms without the use of lasers, but it is extremely inefficient $(\approx 0.1 \%)$, and necessarily results in Ps atoms with energies of many eV. The present experiments were performed using much slower Ps atoms ( $\approx 50 \mathrm{meV}[60])$, and are therefore free of many of the systematic errors present in that work. Slower atoms mean that Doppler effects and transit time broadening are less significant, and that lower microwave power could be employed, essentially eliminating ac Stark shifts and power broadening.

Motional Stark shifts can occur for Ps atoms whose trajectories are not parallel to the magnetic field $[61,62]$. Ps atoms are emitted from silica targets similar to that used in this work with transverse root-mean-square speeds of 
TABLE I. List of systematic errors as described in the text.

\begin{tabular}{lc}
\hline \hline Effect & Shift $(\mathrm{kHz})$ \\
\hline Laser alignment & \pm 215 \\
Motional Stark & \\
Stray electric fields $(<1 \mathrm{~V} / \mathrm{cm})$ & $<+7$ \\
Second order Doppler & +1 \\
ac Stark & +7 \\
\hline \hline
\end{tabular}

$v_{y}^{\mathrm{rms}} \approx 10^{7} \mathrm{~cm} / \mathrm{s}$ [52]. In a magnetic field of $B_{z}=100 \mathrm{G}$ this would result in a motional electric field of $10 \mathrm{~V} / \mathrm{cm}$, perpendicular to the magnetic field, and a corresponding Stark shift of $\approx 700 \mathrm{kHz}$ for the $\nu_{0}$ transition. However, while the motional electric field is linear in $B$, the concomitant Stark shift depends on the square of this field, and therefore on $B^{2}$. This means that any such shifts would extrapolate to zero along with the Zeeman shifts (see Fig. 4) and do not contribute to the systematic error of the zerofield transition frequency determination. Moreover, since they are always in the same direction, motional Stark shifts would result in (magnetic-field-dependent) asymmetric line shapes, which were not observed, as indicated by the asymmetry parameters in Fig. 4(c).

Other possible sources of systematic error are second order Doppler shifts $\left[\approx 1 \mathrm{kHz}\right.$, since $\left(v_{y}^{\mathrm{rms}}\right)^{2} / 2 c^{2} \approx$ $\left.5 \times 10^{-8}\right]$, ac Stark shifts $(<7 \mathrm{kHz}$, based on a determination that the microwave power corresponds to $\approx 25 \%$ of the saturation intensity), and Stark shifts due to stray electric fields. The latter arise from electric fields in the waveguide, caused by patch potentials and other (materialdependent) properties, that are inevitably present to some extent (e.g., Refs. [63-65]). Since Rydberg atoms are highly sensitive to electric fields [66] they can be used to probe stray fields near metal surfaces [67]. Based on previous measurements of stray electric fields close to perforated metal surfaces [68], we expect that the average stray electric field experienced by the atoms in the waveguide was $<1 \mathrm{~V} / \mathrm{cm}$, and the resulting Stark shift of the $\nu_{0}$ transition frequency $<7 \mathrm{kHz}$. Localized positron beam induced charging effects in the waveguide are considered to be negligible.

The largest systematic error in the experiment comes from a possible misalignment of the excitation laser with the axis of the waveguide. The laser alignment process resulted in a beam perpendicular to the waveguide to within an angle of $\theta= \pm 2^{\circ}$, giving possible mean transverse Ps speeds due to laser misalignment of $\pm v_{z} \sin (\theta)=$ $\pm 3.5 \times 10^{5} \mathrm{~cm} / \mathrm{s}$, and a concomitant Doppler shift of $\pm 215 \mathrm{kHz}$ for the $\nu_{0}$ transition. The error budget for all known systematic effects is summarized in Table I and is clearly dominated by Doppler shifts due to possible laser misalignment; we take $\pm 230 \mathrm{kHz}$ to represent the total systematic error of the measurement, neglecting the fact

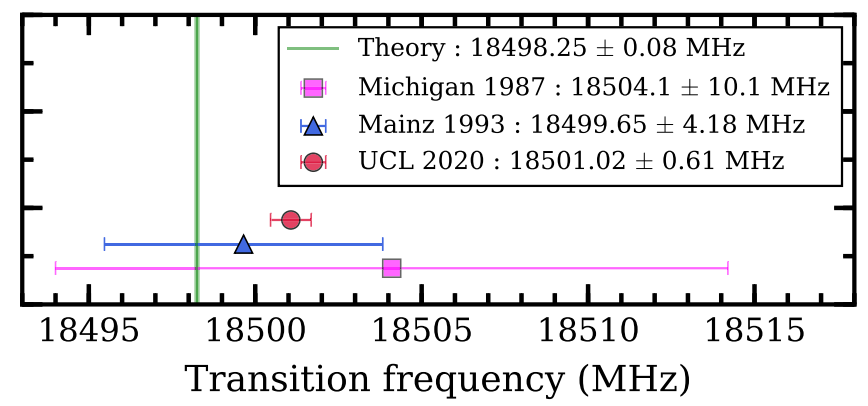

FIG. 5. Previous measurements of the Ps $\nu_{0}$ interval $[35,36]$ and the present result (UCL 2020). The vertical line indicates the theoretical value and uncertainty of the transition frequency [10]. The statistical and systematic errors of the measurements have been added in quadrature.

that all effects other than the laser alignment lead to shifts in only one direction.

The results of all existing $\nu_{0}$ measurements are shown in Fig. 5. The final result of the present experiment is $\nu_{0}=18501.02 \pm 0.57_{\text {stat }} \pm 0.23_{\text {syst }} \mathrm{MHz}$, which represents an almost sevenfold improvement over the quoted precision of the best previous measurement [36]. Despite this increased precision, however, our measurement is not in agreement with the QED calculation of $\nu_{0}=18498.25 \pm 0.08 \mathrm{MHz}$ [10]. The difference amounts to $2.77 \mathrm{MHz}$, or a $4.5 \sigma$ discrepancy. There are no obvious sources of systematic error in the current measurements that could lead to shifts of this magnitude, and the discrepancy therefore warrants further investigation.

There are many improvements that can be made to this experiment to achieve higher precision: (i) extracting the positron beam from the magnetic field [69] would reduce Zeeman shifts to negligible levels. (ii) Using a beam of Rydberg He atoms to measure residual electric fields in the waveguide [5] via microwave spectroscopy (e.g., Ref. [70]) would allow full characterization of any Stark shifts arising from stray fields. (iii) Increasing the $\gamma$-ray detector solid angle coverage could improve count rates by an order of magnitude. (iv) Reversing the direction of propagation of the microwave radiation would allow a direct measurement, and correction, of any Doppler shifts caused by laser misalignment.

The next generation of these experiments will also involve measurements of other fine structure intervals, i.e., the $2^{3} S_{1} \rightarrow 2^{3} P_{1}$ and $2^{3} S_{1} \rightarrow 2^{3} P_{2}$ intervals (see Fig. 3 ). These measurements are more complicated because they are susceptible to interference effects that cause asymmetric line shapes, arising from multiple pathways between initial and final states [53]. These are exacerbated by the substructure of the $2^{3} P_{1}$ and $2^{3} P_{2}$ levels, and larger Zeeman shifts, meaning that accurate determinations of the $\nu_{1}$ and $\nu_{2}$ transition frequencies will likely require magnetic-field-free measurements, and the development of a complete line shape model (e.g., Refs. [71-73]). Line shifts due to interference 
effects that do not result in asymmetric line shapes are possible, but are an order of magnitude too small to explain the present data [74]. To achieve the highest possible precision, it may be beneficial to employ alternative methods that do not require direct knowledge of the line shape (e.g., Refs. $[73,75,76])$.

A longer term goal of this work is to measure the Ps fine structure intervals with uncertainties on the order of $10 \mathrm{kHz}$; this would be equivalent to a part in 5000 of the natural linewidth which, while not unprecedented (e.g., Ref. [71]), cannot be achieved without significant improvements to the experimental methodology. In addition to testing bound state QED theory [6], precision spectroscopy of the Ps fine structure at this level would be sensitive to the ultrafine splitting described by Lamm [39], and could also address tests of spatial noncommutativity [77]. Critically, however, performing measurements with uncertainties commensurate with existing QED theory is a necessary prerequisite for any experimental program designed to search for new physics using precision Ps spectroscopy.

We gratefully acknowledge L. Liszkay for providing silica samples, G. S. Adkins, G. Gribakin, A. P. Mills, Jr., and J. Pérez-Ríos, for helpful discussions, J. Dumper, R. Jawad, and A. Morgan for technical assistance, and R. E. Sheldon and A. M. Alonso for contributions to preliminary stages of this research. This work was supported by the EPSRC under Grant No. EP/R006474/1.

[1] M. K. Gaillard, P. D. Grannis, and F. J. Sciulli, The standard model of particle physics, Rev. Mod. Phys. 71, S96 (1999).

[2] L. Boyle, K. Finn, and N. Turok, CPT-Symmetric Universe, Phys. Rev. Lett. 121, 251301 (2018).

[3] M. S. Safronova, D. Budker, D. DeMille, D. F. Jackson Kimball, A. Derevianko, and C. W. Clark, Search for new physics with atoms and molecules, Rev. Mod. Phys. 90, 025008 (2018).

[4] J. R. Danielson, D. H. E. Dubin, R. G. Greaves, and C. M. Surko, Plasma and trap-based techniques for science with positrons, Rev. Mod. Phys. 87, 247 (2015).

[5] D. B. Cassidy, Experimental progress in positronium laser physics, Eur. Phys. J. D 72, 53 (2018).

[6] S. G. Karshenboim, Precision study of positronium: testing bound state QED theory, Int. J. Mod. Phys. A 19, 3879 (2004).

[7] S. G. Karshenboim, Precision physics of simple atoms: QED tests, nuclear structure and fundamental constants, Phys. Rep. 422, 1 (2005).

[8] J. Govaerts and M. Van Caillie, Neutrino decay of positronium in the standard model and beyond, Phys. Lett. B 381, 451 (1996).

[9] K. Pachucki and S. G. Karshenboim, Complete Results for Positronium Energy Levels at Order $m \alpha^{6}$, Phys. Rev. Lett. 80, 2101 (1998).

[10] A. Czarnecki, K. Melnikov, and A. Yelkhovsky, Positronium $S$-state spectrum: Analytic results at $o\left(m \alpha^{6}\right)$, Phys. Rev. A 59, 4316 (1999).
[11] A. Czarnecki, K. Melnikov, and A. Yelkhovsky, Positronium Hyperfine Splitting: Analytical Value at $o\left(m \alpha^{6}\right)$, Phys. Rev. Lett. 82, 311 (1999).

[12] G. S. Adkins, R. N. Fell, and P. M. Mitrikov, Calculation of the positronium hyperfine interval using the bethe-salpeter formalism, Phys. Rev. A 65, 042103 (2002).

[13] J. Zatorski, $o\left(m \alpha^{6}\right)$ corrections to energy levels of positronium with nonvanishing orbital angular momentum, Phys. Rev. A 78, 032103 (2008).

[14] K. Pachucki and S. G. Karshenboim, Higher-order recoil corrections to energy levels of two-body systems, Phys. Rev. A 60, 2792 (1999).

[15] B. A. Kniehl and A. A. Penin, Order $\alpha^{7} \ln (1 / \alpha)$ Contribution to Positronium Hyperfine Splitting, Phys. Rev. Lett. 85, 5094 (2000).

[16] K. Melnikov and A. Yelkhovsky, $\mathrm{O}\left(\alpha^{3} \ln \alpha\right)$ Corrections to Muonium and Positronium Hyperfine Splitting, Phys. Rev. Lett. 86, 1498 (2001)

[17] M. Baker, P. Marquard, A. A. Penin, J. Piclum, and M. Steinhauser, Hyperfine Splitting in Positronium to $\mathcal{O}\left(\alpha^{7} m_{e}\right)$ : One Photon Annihilation Contribution, Phys. Rev. Lett. 112, 120407 (2014).

[18] G. S. Adkins, M. Kim, C. Parsons, and R. N. Fell, ThreePhoton-Annihilation Contributions to Positronium Energies at Order $m \alpha^{7}$, Phys. Rev. Lett. 115, 233401 (2015).

[19] G. S. Adkins, L. M. Tran, and R. Wang, Positronium energy levels at order $m \alpha^{7}$ : Product contributions in the two-photonannihilation channel, Phys. Rev. A 93, 052511 (2016).

[20] M. I. Eides and V. A. Shelyuto, One more hard three-loop correction to parapositronium energy levels, Phys. Rev. D 96, 011301(R) (2017).

[21] G. S. Adkins, Higher order corrections to positronium energy levels, J. Phys. Conf. Ser. 1138, 012005 (2018).

[22] R. Ley, Atomic physics of positronium with intense slow positron beams, Appl. Surf. Sci. 194, 301 (2002).

[23] A. H. Al-Ramadhan and D. W. Gidley, New Precision Measurement of the Decay Rate of Singlet Positronium, Phys. Rev. Lett. 72, 1632 (1994).

[24] R. S. Vallery, P. W. Zitzewitz, and D. W. Gidley, Resolution of the Orthopositronium-Lifetime Puzzle, Phys. Rev. Lett. 90, 203402 (2003).

[25] O. Jinnouchi, S. Asai, and T. Kobayashi, Precision measurement of orthopositronium decay rate using $\mathrm{SiO}_{2}$ powder, Phys. Lett. B 572, 117 (2003).

[26] M. Deutsch and S. C. Brown, Zeeman effect and hyperfine splitting of positronium, Phys. Rev. 85, 1047 (1952).

[27] A. P. Mills, Jr. and G. H. Bearman, New Measurement of the Positronium Hyperfine Interval, Phys. Rev. Lett. 34, 246 (1975).

[28] M. W. Ritter, P. O. Egan, V. W. Hughes, and K. A. Woodle, Precision determination of the hyperfine-structure interval in the ground state of positronium. V, Phys. Rev. A 30, 1331 (1984).

[29] A. Ishida, T. Namba, S. Asai, T. Kobayashi, H. Saito, M. Yoshida, K. Tanaka, and A. Yamamoto, New precision measurement of hyperfine splitting of positronium, Phys. Lett. B 734, 338 (2014).

[30] S. Chu and A. P. Mills, Jr., Excitation of the Positronium $1^{3} S_{1} \rightarrow 2^{3} S_{1}$ Two-Photon Transition, Phys. Rev. Lett. 48, 1333 (1982). 
[31] S. Chu, A. P. Mills, Jr., and J. L. Hall, Measurement of the Positronium $1^{3} S_{1} \rightarrow 2^{3} S_{1}$ Interval by Doppler-Free TwoPhoton Spectroscopy, Phys. Rev. Lett. 52, 1689 (1984).

[32] M. S. Fee, A. P. Mills, Jr., S. Chu, E. D. Shaw, K. Danzmann, R. J. Chichester, and D. M. Zuckerman, Measurement of the Positronium $1^{3} S_{1} \rightarrow 2^{3} S_{1}$ Interval by Continuous-Wave TwoPhoton Excitation, Phys. Rev. Lett. 70, 1397 (1993).

[33] M. S. Fee, S. Chu, A. P. Mills, Jr., R. J. Chichester, D. M. Zuckerman, E. D. Shaw, and K. Danzmann, Measurement of the positronium $1^{3} S_{1} \rightarrow 2^{3} S_{1}$ interval by continuouswave two-photon excitation, Phys. Rev. A 48, 192 (1993).

[34] A. P. Mills, Jr., S. Berko, and K. F. Canter, Fine-Structure Measurement in the First Excited State of Positronium, Phys. Rev. Lett. 34, 1541 (1975).

[35] S. Hatamian, R. S. Conti, and A. Rich, Measurements of the $2^{3} S_{1}-2^{3} P_{J} \quad(J=0,1,2)$ Fine-Structure Splittings in Positronium, Phys. Rev. Lett. 58, 1833 (1987).

[36] D. Hagena, R. Ley, D. Weil, G. Werth, W. Arnold, and H. Schneider, Precise Measurement of $n=2$ Positronium Fine-Structure Intervals, Phys. Rev. Lett. 71, 2887 (1993).

[37] R. S. Conti, S. Hatamian, L. Lapidus, A. Rich, and M. Skalsey, Search for C-violating, P-conserving interactions and observation of $2^{3} \mathrm{~S}_{1}$ to $2^{1} \mathrm{P}_{1}$ transitions in positronium, Phys. Lett. A 177, 43 (1993).

[38] Th. Udem, R. Holzwarth, and T. W. Hansch, Optical frequency metrology, Nature (London) 416, 233 (2002).

[39] H. Lamm, $p$-state positronium for precision physics: An ultrafine splitting at $\alpha^{6}$, Phys. Rev. A 96, 022515 (2017).

[40] C. Delaunay, C. Frugiuele, E. Fuchs, and Y. Soreq, Probing new spin-independent interactions through precision spectroscopy in atoms with few electrons, Phys. Rev. D 96, 115002 (2017).

[41] Y. V. Stadnik, Probing Long-Range Neutrino-Mediated Forces with Atomic and Nuclear Spectroscopy, Phys. Rev. Lett. 120, 223202 (2018).

[42] C. Frugiuele, J. Pérez-Ríos, and C. Peset, Current and future perspectives of positronium and muonium spectroscopy as dark sectors probe, Phys. Rev. D 100, 015010 (2019).

[43] A. M. Alonso, S. D. Hogan, and D. B. Cassidy, Production of $2^{3} S_{1}$ positronium atoms by single-photon excitation in an electric field, Phys. Rev. A 95, 033408 (2017).

[44] B. S. Cooper, A. M. Alonso, A. Deller, T. E. Wall, and D. B. Cassidy, A trap-based pulsed positron beam optimised for positronium laser spectroscopy, Rev. Sci. Instrum. 86, 103101 (2015).

[45] A. M. Alonso, B. S. Cooper, A. Deller, and D. B. Cassidy, Single-shot positron annihilation lifetime spectroscopy with LYSO scintillators, Nucl. Instrum. Methods Phys. Res., Sect. A 828, 163 (2016).

[46] L. Liszkay, C. Corbel, P. Perez, P. Desgardin, M. F. Barthe, T. Ohdaira, R. Suzuki, P. Crivelli, U. Gendotti, A. Rubbia, M. Etienne, and A. Walcarius, Positronium reemission yield from mesostructured silica films, Appl. Phys. Lett. 92, 063114 (2008).

[47] H. A. Bethe and E. E. Salpeter, Quantum Mechanics of One- and Two-Electron Atoms (Springer, Berlin, 1957).

[48] A. Ore and J. L. Powell, Three-photon annihilation of an electron-positron pair, Phys. Rev. 75, 1696 (1949).

[49] A. M. Alonso, B. S. Cooper, A. Deller, S. D. Hogan, and D. B. Cassidy, Positronium decay from $n=2$ states in electric and magnetic fields, Phys. Rev. A 93, 012506 (2016).

[50] D. B. Cassidy, S. H. M. Deng, H. K. M. Tanaka, and A. P. Mills, Jr., Single shot positron annihilation lifetime spectroscopy, Appl. Phys. Lett. 88, 194105 (2006).

[51] W. Demtröder, Laser Spectroscopy, 3rd ed. (Springer, New York, 2003).

[52] M. H. Rayment, L. Gurung, R. E. Sheldon, S. D. Hogan, and D. B. Cassidy, Multiring electrostatic guide for Rydberg positronium, Phys. Rev. A 100, 013410 (2019).

[53] U. Fano, Effects of configuration interaction on intensities and phase shifts, Phys. Rev. 124, 1866 (1961).

[54] A. E. Miroshnichenko, S. Flach, and Y.S. Kivshar, Fano resonances in nanoscale structures, Rev. Mod. Phys. 82, 2257 (2010).

[55] K. F. Canter, A. P. Mills, Jr., and S. Berko, Observations of Positronium Lyman- $\alpha$ Radiation, Phys. Rev. Lett. 34, 177 (1975).

[56] R Ley, K D Niebling, R Schwarz, and G Werth, Evidence from $n=2$ fine structure transitions for the production of fast excited state positronium, J. Phys. B 23, 1915 (1990).

[57] T. D. Steiger and R. S. Conti, Formation of $n=2$ positronium from untreated metal surfaces, Phys. Rev. A 45, 2744 (1992).

[58] D. C. Schoepf, S. Berko, K. F. Canter, and P. Sferlazzo, Observation of $\operatorname{Ps}(n=2)$ from well-characterized metal surfaces in ultrahigh vacuum, Phys. Rev. A 45, 1407 (1992).

[59] D. J. Day, M. Charlton, and G. Laricchia, On the formation of excited state positronium in vacuum by positron impact on untreated surfaces, J. Phys. B 34, 3617 (2001).

[60] D. B. Cassidy, P. Crivelli, T. H. Hisakado, L. Liszkay, V. E. Meligne, P. Perez, H.W.K. Tom, and A. P. Mills, Jr., Positronium cooling in porous silica measured via Doppler spectroscopy, Phys. Rev. A 81, 012715 (2010).

[61] S. M. Curry, Combined Zeeman and motional stark effects in the first excited state of positronium, Phys. Rev. A 7, 447 (1973).

[62] C. D. Dermer and J. C. Weisheit, Perturbative analysis of simultaneous Stark and Zeeman effects on $n=1 \rightarrow n=2$ radiative transitions in positronium, Phys. Rev. A 40, 5526 (1989).

[63] T. W. Darling, F. Rossi, G. I. Opat, and G. F. Moorhead, The fall of charged particles under gravity: A study of experimental problems, Rev. Mod. Phys. 64, 237 (1992).

[64] A. Härter, A. Krükow, A. Brunner, and J. Hecker Denschlag, Long-term drifts of stray electric fields in a Paul trap, Appl. Phys. B 114, 275 (2014).

[65] J. L. Garrett, D. Somers, and J. N. Munday, The effect of patch potentials in casimir force measurements determined by heterodyne kelvin probe force microscopy, J. Phys. Condens. Matter 27, 214012 (2015).

[66] T.F. Gallagher, Rydberg Atoms (Cambridge University Press, Cambridge, England, 1994).

[67] A. Osterwalder and F. Merkt, Using High Rydberg States as Electric Field Sensors, Phys. Rev. Lett. 82, 1831 (1999).

[68] S. D. Hogan, J. A. Agner, F. Merkt, T. Thiele, S. Filipp, and A. Wallraff, Driving Rydberg-Rydberg Transitions from a Coplanar Microwave Waveguide, Phys. Rev. Lett. 108, 063004 (2012). 
[69] T. R. Weber, J. R. Danielson, and C. M. Surko, Electrostatic beams from tailored plasmas in a Penning-Malmberg trap, Phys. Plasmas 17, 123507 (2010).

[70] A. Deller and S. D. Hogan, Microwave spectroscopy of the 1 sn $p^{3} P_{J}$ fine structure of high Rydberg states in ${ }^{4} \mathrm{He}$, Phys. Rev. A 97, 012505 (2018).

[71] A. Beyer, L. Maisenbacher, A. Matveev, R. Pohl, K. Khabarova, A. Grinin, T. Lamour, D. C. Yost, T. W. Hänsch, N. Kolachevsky, and T. Udem, The Rydberg constant and proton size from atomic hydrogen, Science 358, 79 (2017).

[72] R. C. Brown, S. Wu, J. V. Porto, C. J. Sansonetti, C. E. Simien, S. M. Brewer, J. N. Tan, and J. D. Gillaspy, Quantum interference and light polarization effects in unresolvable atomic lines: Application to a precise measurement of the ${ }^{6,7} \mathrm{Li} D_{2}$ lines, Phys. Rev. A 87, 032504 (2013).

[73] N. Bezginov, T. Valdez, M. Horbatsch, A. Marsman, A. C. Vutha, and E. A. Hessels, A measurement of the atomic hydrogen lamb shift and the proton charge radius, Science 365, 1007 (2019).

[74] A. Marsman, M. Horbatsch, and E. A. Hessels, The effect of quantum-mechanical interference on precise measurements of the $n=2$ triplet $\mathrm{P}$ fine structure of helium, J. Phys. Chem. Ref. Data 44, 031207 (2015).

[75] N. F. Ramsey, Experiments with separated oscillatory fields and hydrogen masers, Rev. Mod. Phys. 62, 541 (1990).

[76] K. Kato, T. D. G. Skinner, and E. A. Hessels, UltrahighPrecision Measurement of the $n=2$ Triplet $P$ Fine Structure of Atomic Helium Using Frequency-Offset Separated Oscillatory Fields, Phys. Rev. Lett. 121, 143002 (2018).

[77] M. Haghighat, S. M. Zebarjad, and F. Loran, Positronium hyperfine splitting in noncommutative space at order $\alpha^{6}$, Phys. Rev. D 66, 016005 (2002). 